\title{
Near-Milne realization of scale-invariant fluctuations
}

\author{
João Magueijo ${ }^{1,2,3}$ \\ ${ }^{1}$ Perimeter Institute for Theoretical Physics, 31 Caroline St N, Waterloo N2L 2Y5, Canada \\ ${ }^{2}$ Canadian Institute for Theoretical Astrophysics, 60 St George St, Toronto M5S 3H8, Canada \\ 3 Theoretical Physics Group, Imperial College, Prince Consort Road, London SW7 2BZ, England
}

\begin{abstract}
A near-Milne Universe produces a very red spectrum of vacuum quantum fluctuations, but has the potential to produce near-scale invariant thermal fluctuations. This happens if the energy and entropy are mildly sub-extensive, for example if there is a Casimir contribution. Therefore, one does not need to invoke corrections to Einstein gravity (as in loop quantum cosmology) for a thermal scenario to be viable. Neither do we need the energy to scale like the area, as in scenarios where the thermal fluctuations are subject to a phase transition in the early Universe. Some odd features of this model are pointed out: whether they are fatal or merely unusual should be the subject of future investigations.
\end{abstract}

The near scale-invariance of the primordial density fluctuations has become a central fact in modern cosmology and is now well-established by observation [1]. It is still debatable whether deviations from exact scaleinvariance have been observed (see, e.g. [2, 3]), and to what significance level. But the spectral index $n_{S}$ cannot be substantially different from 1 , if it differs from 1 at all. The possibility that these fluctuations might have a thermal origin has long been entertained [4, 5, [6]. In this paper we propose a new thermal scenario that leads to (quasi) scale-invariance.

Thermal scenarios require new physics in order to produce near scale-invariant fluctuations. As shown in [7], and reviewed later in this paper, if one merely modifies the equation of state of thermal matter it is found that in general the spectral index is either $n_{S}=4$ (if the modes are dynamically extracted from a sub-Hubble thermal bath) or $n_{S}=0$ (if there is a phase transition, so that the final spectrum is proportional to the equaltemperature spectrum). Therefore, prima facie, thermal fluctuations lead to spectra that are either too blue or too red. Independently of this result, we shall call these two types of scenario Type A (no phase transition) and $\mathrm{B}$ (phase transition).

New physics, naturally, may come to the rescue, modifying the standard calculation and rendering thermal scenarios viable. Although this feature necessarily makes them more unconventional, it is also precisely why they may be an interesting gateway into phenomenological quantum gravity and string theory. For example, in type A scenarios, loop quantum cosmology [8] is known to introduce enough novelties in the gravitational sector to allow for thermal fluctuations to produce (quasi) scaleinvariance [7]. In Type B scenarios, where there is a phase transition "photographing" the equal-temperature spectrum, one needs to deform the matter sector instead. If the energy becomes strongly non-extensive, specifically proportional to the area, then the equal temperature thermal spectrum is scale-invariant. This might happen in an holographic phase of loop quantum gravity [10] or in the Hagedorn phase for certain types of string gas [9].

In this paper we explore an intermediate possibility, combining a type A scenario (where the modes imprinted outside the horizon froze-in at different temperatures), with the main feature required to render type B models viable: non-extensiveness of the energy. This allows us to considerably soften the assumptions about new physics. In contrast with the work of [7], we assume that the Einstein equations are not modified, to zeroth or first order. In analogy with the work of [9, 10] we let the energy and entropy be non-extensive; but we find that they need only be mildly sub-extensive for near scale-invariance to be achieved, near the Milne point, $w \approx=-1 / 3$.

The case against thermal fluctuations at first seems utterly damning. It is well known that thermal matter does not need to have the usual equation of state $w=1 / 3$. For example deformed dispersion relations have been discussed that induce a variety of $w=p / \rho$, in particular $w<-1 / 3$. One can then have scenarios where the modes start inside the horizon, in a thermal state, and then are pushed out of the horizon, where they fall out of thermal equilibrium. We can consider either contracting Universes with $w>-1 / 3$, or expanding Universes with $w<-1 / 3$. The question is then what is the spectrum of such fluctuations, as imprinted outside the horizon?

The standard lore is to start by working out the fluctuations inside the horizon, considering a generic box of volume $V$. From the partition function

$$
Z=\sum_{r} e^{-\beta E_{r}}
$$

(where $\beta=T^{-1}$ ) we find that the total energy $U$ inside volume $V$ is given by:

$$
U=\langle E\rangle=\frac{\sum_{r} E_{r} e^{-\beta E_{r}}}{\sum_{r} e^{-\beta E_{r}}}=-\frac{d \log Z}{d \beta}
$$

whereas its variance is given by

$$
\sigma_{E}^{2}=\left\langle E^{2}\right\rangle-\langle E\rangle^{2}=\frac{d^{2} \log Z}{d \beta^{2}}=-\frac{d U}{d \beta}=T^{2} c_{V}
$$

where $c_{V}$ is the specific heat at constant volume. This establishes a very simple relation between thermal fluctuations and the specific heat at constant volume, and this 
beautiful relation has been known since the XIX century, and is textbook material [24].

It is more common to discuss fluctuations in Fourier space. This can be achieved by noting the proportionality between the variance $\sigma_{g}^{2}(R)$ (in any quantity $g$ ) defined in position space and smeared on a scale $R$, and the "dimensionless power spectrum" $\mathcal{P}_{g}$. Specifically $\sigma_{g}^{2}(R)=\left\langle\delta g^{2}\right\rangle_{R} \approx \mathcal{P}_{g}\left(k_{R}=a / R\right)$. This formula has been questioned in some regimes [25, 26], but we shall stay well away from the problem cases. Then, using (3) we have

$$
\mathcal{P}_{\delta \rho}(k) \sim\left\langle\delta \rho^{2}\right\rangle_{R=\frac{a}{k}}=\frac{1}{R^{6}}\left\langle\delta E^{2}\right\rangle_{R}=\frac{\sigma_{E}^{2}(R)}{R^{6}}=\frac{T^{2}}{R^{6}} c_{V} .
$$

We must now find out the associated metric fluctuations. The most robust description is in terms of the (spatial) curvature perturbation in the comoving gauge, $\zeta$. However, the Newtonian potential in the longitudinal gauge, $\Phi$, is also commonly in use. Outside the horizon, $\zeta$ is proportional to $\Phi$ in expanding Universes with $-5 / 3<w<1$. In this regime they both "freeze-in", have the same spectrum and can be used interchangeably. Specifically $\zeta \approx 2 \Phi$ near the Milne point to be studied in this paper.

In finding the potential/curvature left outside the horizon we must compute it from the matter fluctuations at horizon crossing. Depending on which gauge one uses the relevant expressions look different, but they all lead to the same result: at horizon crossing the potential is proportional to the density contrast $\delta=\delta \rho / \rho$. The proportionality constant differs by a factor of order one but the spectral index is always the same. For example in the longitudinal gauge the Hamiltonian constraint gives:

$$
k^{2} \Phi+3 H(\dot{\Phi}+H \Phi)=4 \pi G a^{2} \delta \rho^{l . g} .
$$

whereas using the comoving density we find

$$
k^{2} \Phi=4 \pi G a^{2} \delta \rho^{c . g},
$$

(see Eqn. 14.151 of [13] or Eqn. 4.3 of [14]). Whichever of these expressions one uses for $\Phi$ at horizon crossing (defined as $k \sim a H$ or $k \eta \sim 1$ ) the result:

$$
\mathcal{P}_{\Phi} \sim \frac{a^{4} \mathcal{P}_{\delta \rho}}{k^{4}} \sim \frac{a^{4}}{k^{4}}\left[\frac{T^{2}}{R^{6}} c_{V}\right]_{R=\frac{a}{k}}=\frac{k^{2}}{a^{2}} T^{2}\left[c_{V}\right]_{R=\frac{a}{k}}
$$

If we now assume the extensive nature of the energy we have $c_{V}=\rho^{\prime}(T) R^{3}$ (with $\rho^{\prime}=d \rho / d T$ ), so finally

$$
\mathcal{P}_{\Phi} \sim \frac{a}{k} T^{2} \rho^{\prime},
$$

that is, the fluctuations at a fixed temperature are white noise $\left(n_{S}=0\right)$, and have an amplitude that only depends on the Stephan-Boltzmann law, relating energy density and temperature. We shall assume that $\rho \propto T^{\zeta}$, where $\zeta$ is a parameter.
As the $\Phi$ modes leave the horizon their amplitude gets fixed at whatever thermal amplitude they have at crossing, that is:

$$
\mathcal{P}_{\Phi}(k) \sim\left[\frac{a}{k} T^{2} \rho^{\prime}\right]_{k=a H} .
$$

Using the Friedman equation $H^{2} \propto \rho$ we therefore have

$$
\mathcal{P}_{\Phi}(k) \sim\left[\frac{T^{2} \rho^{\prime}}{\sqrt{\rho}}\right]_{k=a H}
$$

where $k=a H$ specifies a relation between a given comoving $k$ leaving the horizon at a given time, and the temperature, thereby allowing the inversion of the right hand side as a function of $k$. Eqn. (10) implies:

$$
\frac{d \ln \mathcal{P}_{\Phi}}{d \ln T}=1+\frac{\zeta}{2}
$$

The relation between $k=a H$ and the temperature, however, depends on both the equation of state $p=w \rho$ and $\rho \propto T^{\zeta}$. Using the Friedmann equation we have $k=a H \propto a \sqrt{\rho}$, and since $\rho \propto 1 / a^{3(1+w)}$, we may derive

$$
a \propto T^{\frac{-\zeta}{3(1+w)}} .
$$

Therefore:

$$
\frac{d \ln k}{d \ln T}=\frac{-\zeta}{3(1+w)}+\frac{\zeta}{2}=\frac{\zeta(1+3 w)}{6(1+w)}
$$

and the spectral index is

$$
n_{S}-1=\frac{d \ln \mathcal{P}_{\Phi}}{d \ln k}=\frac{d \ln \mathcal{P}_{\Phi}}{d \ln T} \frac{d \ln T}{d \ln k}=3 \frac{2+\zeta}{\zeta} \frac{1+w}{1+3 w}
$$

A similar result can be obtained by considering the outside and inside the horizon expressions for $\Phi$ and matching them at $k \eta \sim 1$ (as in the heuristic argument presented at the start of [15]).

Immediately two interesting candidates for scale invariance stand out. Firstly $\zeta=-2$, that is $\rho \propto 1 / T^{2}$; this may lead to scale-invariance because the amplitude of the frozen-in thermal fluctuations does not depend on the temperature when they leave the horizon (c.f. Eqn 10). Secondly $w=-1$ : this could lead to scale-invariance because $\rho$ does not change, and so neither does the temperature or amplitude of the fluctuations as they leave the horizon. This is the scenario studied in [11, 12].

However further conditions apply. It's been noted 21, 22, 23] that the equation of state $p=w \rho$ and the Stephan-Boltmann law $\rho=\rho(T)$ are linked by a thermodynamical relation. The argument assumes that energy and entropy are extensive. Consider the first law of thermodynamics:

$$
d U=-p d V+T d S
$$

If the energy $U$ and entropy $S$ are extensive, then $U(\lambda V, \lambda S)=\lambda U(V, S)$. Taking a derivative with respect 
to $\lambda$ at $\lambda=1$, and using (15) we arrive at the Euler relation

$$
U=-p V+T S
$$

so that defining $\rho=U / V$ and $s=S / V$ we have

$$
s=\frac{p+\rho}{T}
$$

We can now prove that $s=d p / d T$ in a variety of ways, e.g. introducing the free energy $F=U-T S=F(V, T)$, so that $d F=-p d V-S d T$. This leads to the integrability condition:

$$
s=\left(\frac{\partial S}{\partial V}\right)_{T}=\left(\frac{\partial p}{\partial T}\right)_{V}
$$

Thus the expression

$$
\frac{d p}{d T}=\frac{p+\rho}{T}
$$

If $w$ is a constant we obtain that $\rho \propto T^{\zeta}$ with

$$
\zeta=1+\frac{1}{w}
$$

This relation implies that $\zeta=0$ for $w=-1$ : "deformed" radiation may behave like a cosmological constant, but then the specific heat vanishes and there are no thermal fluctuations at all. For $\zeta=-2$ it leads to $w=-1 / 3$, that is, exactly a Milne Universe. This is merely a borderline case for solving to the horizon problem: the comoving horizon does not increase but neither does it increase. In fact this relation implies that $n_{s}=4$ for all values of $w$ and clearly it must be broken if this type of scenario is to be viable.

This can be achieved if the energy and entropy are subextensive as we now prove. Let's suppose that energy and entropy they scale like

$$
\begin{aligned}
U & =\rho_{C}(T) V^{1-\gamma} \\
S & =s_{C}(T) V^{1-\gamma}
\end{aligned}
$$

with $\gamma>0$. Then the Euler relation (16) is modified, creating a parallel with the Casimir energy, as pointed out in [21]. Specifically, we can prove, along the same lines as before, that now

$$
(1-\gamma) s_{C}=\frac{p_{C}+\rho_{C}(1-\gamma)}{T}
$$

with pressure defined as $p_{C}(T)=p V^{\gamma}$. The Casimir energy defined for this model is

$$
E_{C}=3(U+p V-T S)=3 \gamma F
$$

where $F=U-T S$ is the free energy. As before, using $d F=-p d V-S d T$ we can prove that

$$
\frac{d p_{C}}{d T}=(1-\gamma) s_{C}
$$

and this now leads to

$$
\frac{d p_{C}}{d T}=\frac{p_{C}+\rho_{C}(1-\gamma)}{T}
$$

For $p_{C}=w_{C} \rho_{C}$ and $\rho_{C} \propto T^{\zeta_{C}}$ we therefore have

$$
\zeta_{C}=1+\frac{1-\gamma}{w_{C}}
$$

a constraint to be contrasted with (20).

This modification is sufficient to break both the whitenoise nature of the spectrum at fixed temperature, and the conclusion that $n_{S}=4$ if we extract modes at different temperatures. Specifically the equal-time power spectrum is

$$
\mathcal{P}_{\Phi}=T^{2} \rho_{C}^{\prime}\left(\frac{a}{k}\right)^{1-3 \gamma}
$$

and we see that for $\gamma=2 / 3$ the (equal time) spectrum is scale-invariant, as derived in the holographic scenario of [10].

For more modest values of $\gamma$ the spectrum is only slightly non-white. However we can now find a condition for scale invariance outside the horizon. If the modes freeze for $k=a H$ and if the Einstein equations (zero and first order) are unmodified, then outside the horizon we have

$$
\frac{d \ln \mathcal{P}_{\Phi}}{d \ln T}=1+\frac{\zeta_{C}}{2}(1+3 \gamma)
$$

so that the condition for scale-invariance is

$$
\zeta_{C}=\frac{-2}{1+3 \gamma} .
$$

Making use of the modified constraint (27) this translates into

$$
w_{C}=-\frac{(1-\gamma)(1+3 \gamma)}{3(1+\gamma)} \approx-\frac{1}{3}(1+\gamma)
$$

so that for $\gamma>0$ (sub-extensive energy) we can have $w<$ $-1 / 3$. Naturally we can also fix the parameter to make the spectrum as close or deviant from scale-invariance as required, with result

$$
n_{S}-1=3 \frac{1+\gamma \frac{3\left(w_{C}-\gamma\right)-4}{1+3 w_{C}}}{1-\frac{\gamma}{1+w_{C}}}
$$

What might go wrong with such a scenario? Most obviously there is the issue of the validity of the whole set up. If $w<0$, then $\zeta<0$. If $T>0$ (and $\rho>0$ ) this would entail a negative specific heat $c_{V}$, something that signals the break down of the canonical ensemble and the onset of a phase transition(see, e.g. [17]). Indeed expression (3) is the standard proof for the fact that the specific heat must be positive [18]. This conclusion, however, can be bypassed if we are prepared to accept negative temperatures [19]. Negative temperatures have been discussed in 
the context of nuclear spin systems, and the usual formulae of thermodynamics and statistical physics can be easily adapted to them [16]. Much the same arguments that can be made with phantom matter, then, apply here [19].

Perhaps the strongest constraint on this type of scenario may derive from the number of e-foldings required to produce the whole observable Universe. If $w$ is very close to $-1 / 3$ this can be vastly larger than in the deSitter case, and since $H$ and $T$ are varying obvious constraints ensue, telling us how far we must be from the Milne Universe, that is how large $|w+1 / 3|$ must be. This translates into a constraint on the necessary $\gamma$ (see Eqn. (31) and (32) ), so that the sub-extensiveness may not be mild at all. If if this is the case, however, we argue that these scenarios are still worth pursuing.

To summarize we have investigated a thermal scenario that leads to near scale-invariant fluctuations. It bypasses the usual result $n_{S}=4$ by allowing the energy and entropy to be mildly sub-extensive. Corrections to Einstein's gravity do not need to be invoked if $w$ is smaller than $-1 / 3$, but not by much. Neither does the energy need to go like the area, as in other (more speculative) work. Indeed we advocate this model as the most conservative viable thermal scenario in existence. It is curious that this region of parameter space, so unsuitable for the vacuum fluctuations (for which, then, $n_{S} \sim-\infty$ ), is so hospitable to thermal fluctuations.

There are other "conservative" ways in which the standard $n_{S}=4$ result may be avoided. If we accept a two fluid system, one responsible for the fluctuations, the other for the background evolution, then a viable scenario also follows without any extra assumptions. However such scenarios are extremely fine tuned, as we shall detail in a future publication. The scenario presented here may fare much better, with only the simple extra assumption of a Casimir contribution to the energy of the Universe.

I'd like to thank Robert Brandenberger, Parampreet Singh and Andrew Tolley for helpful discussions. Research at PI is supported in part by the Government of Canada through NSERC and by the Province of Ontario through MEDT.
[1] Spergel D., et al., 2006, astro-ph/0603449

[2] J. Magueijo and R. Sorkin, astro-ph/0604410

[3] Liddle A., 2007, astro-ph/0701113

[4] Principles of Physical Cosmology, P. J. E. Peebles, Princeton University Press, New Jersey, 1993.

[5] S. Alexander, J. Magueijo, hep-th/0104093

[6] J. Magueijo and L. Pogosian, Phys. Rev. D67: 043518, 2003.

[7] J. Magueijo and P. Singh, hep-th/0702217

[8] A. Ashtekar, gr-qc/0702030.

[9] A. Nayeri, R. Brandenberger and C. Vafa, Phys. Rev. Lett.97: 021302, 2006; R. Brandenberger et al, hep-th/0608121 and hep-th/0608186; Biswas et al, hep-th/0610274 R. Brandenberger et al, JCAP 0611:009,2006.

[10] J. Magueijo, L. Smolin and C. Contaldi, astro-ph/0611695.

[11] S. Alexander, R. Brandenberger, J. Magueijo, Phys.Rev. D67 (2003) 081301.
[12] S. Koh, R. Brandenberger, hep-th/0702217.

[13] A. Liddle and D. Lyth, Cosmological inflation and largescale structure, CUP 2000.

[14] H. Kodama and M. Sasaki, Prog. Th. Phys. 78 (1984) 1.

[15] A. Tolley and D. Wesley, hep-th/0703101.

[16] N. Ramsey, Phys. Rev. 103 (1956) 20.

[17] D. Lynden-Bell, cond-mat/9812172.

[18] E. Schrödinger, Statistical Thermodynamics, Cambridge, 1952.

[19] P. Gonz"alez-Díaz and C. Sigüenza, astro-ph/0407421

[20] P. Singh, Class. Quant. Grav. 22, 4203 (2005).

[21] E. Verlinde, hep-th/0008140

[22] D. Youm, hep-th/0201268

[23] P. Gonzales-Dias and C. Siguenza, astro-ph/0407421.

[24] C. Kittel, Thermal Physics, John Wiley and Sons, New York, 1969.

[25] A. Gabrielli, M. Joyce, F. Labini, astro-ph/0110451

[26] D. Oaknin, hep-th/0305068; hep-th/0308078. 